\title{
Comparative Study of Autologous Radiocephalic and Brachiocephalic Arteriovenous Fistula in Patients with End Stage Renal Disease
}

\author{
Magar $\mathrm{DT}^{1}$, Shrestha $\mathrm{K}^{1}$, Chapagain $\mathrm{D}^{1}$, Shrestha $\mathrm{K}^{1}$, Thapa $\mathrm{S}^{2}$
}

\section{ABSTRACT}

Introduction: End-stage renal disease requires treatment with dialysis or renal transplantation. For the dialysis, autologous radiocephalic (RC) or brachiocephalic (BC) arteriovenous fistula (AVF) is the better option for vascular access for hemodialysis. Aim: The aim of this study is to find out the outcome between RC AVF and BC AVF. Methods: This is the retrospective study, conducted for the period of 24 months from September 2017 to September 2019 in the department of Cardiothoracic and Vascular Surgery of Bir Hospital, Nepal. RC and BC AVF were created for the assess of hemodialysis. Outcome and different complications were taken into consideration. Results: The total number of patients included in this study was 400 . The overall failure rate of autologous AV fistula was $12.75 \%$. Out of these, the failure rate was more in RC AV fistula group, 34 (17\%) than in BC AV fistula group, 17 (8.5\%). The most common complication was bleeding in both groups having an overall rate of 39 (9.75\%). The limb edema was more common in BC AV fistula group 16 (8.0\%) then in RC AV fistula group 7(3.5\%). The overall infection rate was $4.5 \%$. Overall patency rate was $87.25 \%$.Conclusions: Autologous RC AVF and BC AVF are the choices for vascular access for hemodialysis in patients with end-stage renal disease. BC AVF has a better patency rate than RC AVF but with the slight higher risk of complications.

\section{Keywords: Arteriovenous fistula, Brachiocephalic, Endstage renal disease, Hemodialysis, Patency, Radiocephalic}

\section{Authors:}

1. Dr. Deepak Thapa Magar

2. Dr. Kiran Shrestha

3. Dr. Dinesh Chapagain

4. Dr. Kumar Shrestha

5. Dr. Sara Thapa

${ }^{1}$ Cardiothoracis and Vascular Surgery unit, Department of Surgery, NAMS, Bir hospital

${ }^{2}$ Chitwan Medical College, Chitwan

\section{Address for Correspondence:}

\author{
Dr. Deepak Thapa Magar \\ Chief Consultant Surgeon, CTVS unit, \\ Department of surgery \\ NAMS, Bir Hospital \\ E-mail: deepakthapamagar@gmail.com
}

\section{INTRODUCTION}

End-stage renal disease (ESRD) is a major public health problem, requiring treatment with dialysis or renal transplantation .The incidence of which is increasing every year worldwide. For the approximately 640,000 patients in the United States with end-stage renal disease (ESRD), the majority (64.2\%) undergo renal replacement therapy via hemodialysis. Less frequently used renal replacement therapy treatments are renal transplant (29.3\%) and peritoneal dialysis (6.4\%). ${ }^{1}$ The methods of delivering hemodialysis include tunneled and non-tunneled catheters, arteriovenous grafts (AVGs), and arteriovenous fistulas (AVFs). Central venous hemodialysis catheters, although often necessary, should be avoided whenever possible. Because, among the hemodialysis delivery options, they have the highest rates of thrombosis, infection and central vein stenosis ${ }^{2}$, especially when inserted from a subclavian vein. Blood flow rates from hemodialysis catheters are typically the lowest of all hemodialysis access types. Finally, hemodialysis catheters are associated with greater mortality than AVGs or AVFs. ${ }^{3}$

Arteriovenous fistula has been the vascular access of choice for hemodialysis because of lower cost, morbidity and mortality. ${ }^{4}$ The three principal forms of chronic vascular access for hemodialysis are native arteriovenous fistulas (native AVFs), arteriovenous shunts using graft material (AV graft), and tunneled double-lumen catheters. Of these, the native arteriovenous fistula is preferred for long-term hemodialysis vascular access since it has the best long-term primary patency rate, requires the fewest interventions of any type of access, and most importantly, arteriovenous fistulas are associated with the lowest incidence of morbidity and mortality. ${ }^{5}$ There are three main types of AVFs. The radiocephalic fistula is a 
forearm fistula created by anastomosing the side of a radial artery to the end of a cephalic vein, also referred to as the Brescia-Cimino fistula. The brachiocephalic fistula is an upper arm fistula created by connecting the side of a brachial artery to the end of a cephalic vein at the level of the elbow. Finally, the brachial artery-to-transposed basilic vein (BTB) fistula which is created by anastomosing the side of a brachial artery to the end of a basilic vein that has been transposed laterally and elevated superficially to make it amenable to dialysis cannulation. We have conducted the study with the aim to compare the overall outcomes and complications of radiocephalic and brachiocephalic AVF for hemodialysis in end stage renal disease. The objectives of this study were to compare the outcome and rate of complications in radiocephalic \& brachiocephalic arteriovenous fistula (AVF).

\section{METHODS}

This is the hospital based retrospective study, carried out over the period of 2 years from Sept 2017 to Sept 2019 in CTVS, department of surgery Bir Hospital, Nepal.

Total of 400 patients with ESRD requiring HD who were referred to the CTVS department for access were recruited. Vascular assessment was done by Allen's test, evaluation for venous thrombosis and venous size was performed clinically by palpation, when not appreciable with the help of Doppler studies. In all fistulas autologous veins were used. The radio cephalic fistulas were created using radial artery and cephalic vein end to side anastomosis in a non-dominant hand. When the radial artery and the cephalic veins were not suitable because of small diameter or previously operated, brachiocephalic AVF were created using brachial artery and cephalic vein end to side anastomosis. We excluded patients who had thrombosed cephalic veins, patients with uremic symptoms and with negative Allen's test.

\section{Procedure Details}

After evaluating the patients, all the needed investigations were performed. In the Operation Theater (OT), patients were positioned in supine. Incision site, usually the wrist of a non -dominant hand, was infiltrated with Injection Xylocaine $2 \%$. Then slightly curvilinear incision was given. First cephalic vein was identified and dissected until the adequate length was obtained, then vein ligated and divided distally. Diluted Heparin was infiltrated to flush the vessels. Radial artery was identified, isolated. End to side anastomosis done with continuous Polypropylene 6.0 round body. Similar steps were taken creating the brachiocephalic arteriovenous fistula at elbow. Thrill assessed after the anastomosis, hemostasis secured and wound was closed. After the procedure antibiotic and analgesic were prescribed. Patients were advised to exercise the operated hand with a solid foam-rubber ball the size of a tennis ball (fistula ball) and discharged after the surgery.
Patients were advised to follow up in CTVS OPD after one week and then after 6 weeks to assess the maturation of the fistula. Similarly, patients were evaluated for complications like infection, hematoma, thrombosis, aneurysms and steal syndrome,

\section{Data collection}

Total of 400 cases, 200 in each group of radio cephalic and brachiocephalic fistula were taken for the studies. Approval from Subject Committee and Institutional Review Board of National Academy of Medical Sciences was taken prior to study. Data were collected from the surgery registers.

\section{RESULTS}

The age of the patient ranges from 15 to 81 years, with the mean age $56 \pm 16.12$ years. Male dominant 263 (65.75\%), female 137 (34.25\%). Both RC fistula and BC fistula created in 200 patients. The age group of the patients mostly diagnosed with ESRD was more than 56 years of age. (Table I)

\begin{tabular}{|lcc|}
\hline \multicolumn{1}{|c}{ Variables } & RC AV fistula (200) & BC AV fistula (200) \\
\hline Age & & \\
$15-25$ & 4 & 7 \\
& $(2.0 \%)$ & $(3.5 \%)$ \\
\hline $26-35$ & 27 & 18 \\
& $(13.5 \%)$ & $(9.0 \%)$ \\
\hline $36-45$ & 35 & 27 \\
& $(17.5 \%)$ & $(13.5 \%)$ \\
\hline $56-65$ & 37 & 48 \\
& $(18.5 \%)$ & $(24.0 \%)$ \\
\hline 76 & 53 & 49 \\
\hline Sex & $(26.5 \%)$ & $(24.5 \%)$ \\
\hline Male & 44 & 51 \\
& $(22.0 \%)$ & $(25.5 \%)$ \\
\hline Female & & 111 \\
& 152 & $(55.5 \%)$ \\
\hline
\end{tabular}

Table I : Age Distribution according to Type of Fistula \& Gender

Out of 400 patients 87 (21.75\%) had Diabetes mellitus, having similar numbers in both RC group (22.0\%) and BC group $(21.5 \%)$. But there was a huge difference in number in case of re surgery in two groups. In RC group re surgery was done in $39(19.5 \%)$ cases while in $B C$ group re surgery was done in 73 ( $36.5 \%$ ) cases due to failure of previously created AV fistula. The overall percentage patients who were not on dialysis at the time creating the AV fistula was just $7.5 \%$. ( Table II)

\begin{tabular}{lccc|}
\multicolumn{1}{c}{ Variables } & $\begin{array}{c}\text { RC AV fistula } \\
\mathbf{n}=\mathbf{2 0 0}\end{array}$ & $\begin{array}{c}\text { BC AV fistula } \\
\mathbf{n}=\mathbf{2 0 0}\end{array}$ & Total $\mathbf{n = 4 0 0}$ \\
Diabetes Mellitus & 44 & 43 & 87 \\
& $(22.0 \%)$ & $(21.5 \%)$ & $(21.75 \%)$ \\
Re - surgery & 39 & 73 & 112 \\
& $(19.5 \%)$ & $(36.5 \%)$ & $(28.0 \%)$ \\
Not on dialysis & 21 & 9 & 30 \\
& $(10.5 \%)$ & $(4.5 \%)$ & $(7.5 \%)$
\end{tabular}

Table II : Comparison of comorbidities and re surgery 
Magar et al.: Comparative Study of Autologous Radiocephalic and Brachiocephalic Arteriovenous Fistula in Patients with End Stage Renal Disease

The overall failure rate of autologous AV fistula was $12.75 \%$. Out of these the failure rate was more in RC AV fistula group, $34(17 \%)$ than in BC AV fistula group, 17 (8.5\%). The most common complication was bleeding in both groups having an overall rate of 39 (9.75\%). The limb edema was more common in BC AV fistula group 16 (8.0\%) then in RC AV fistula group, $7(3.5 \%)$. The overall infection rate was $4.5 \%$.

\begin{tabular}{|l|c|c|c|}
\hline Variables & $\begin{array}{c}\mathbf{R C ~ A V} \text { fistula } \\
\mathbf{n = 2 0 0}\end{array}$ & $\begin{array}{c}\mathbf{B C} \text { AV fistula } \\
\mathbf{n = 2 0 0}\end{array}$ & Total $\mathbf{n = 4 0 0}$ \\
\hline Edema & 7 & 16 & 23 \\
& $(3.5 \%)$ & $(8.0 \%)$ & $(5.75 \%)$ \\
\hline Bleeding & 21 & 18 & 39 \\
\hline Infection & $(10.5 \%)$ & $(9.0 \%)$ & $(9.75 \%)$ \\
\hline & 6 & 12 & 18 \\
\hline Failure & $(3.0 \%)$ & $(6.0 \%)$ & $(4.5 \%)$ \\
\hline
\end{tabular}

Table 3 : Complication \& failure of AV Fistula

\section{DISCUSSION}

A progressive rise in the number of patients accepted for renal replacement therapy has been reported worldwide, permanent vascular access is the life-line for the majority of these patients, when hemodialysis is the treatment of choice. Thus, the successful creation of functional permanent vascular access is vital in order to deliver adequate hemodialysis therapy in ESRD. ${ }^{6}$ The AVF is also important for the outcome of a patient on HD as it has a significant impact on survival. ${ }^{7}$

The distal radiocephalic AVF described in 1966 by Brescia et al ${ }^{8}$ is considered the first procedure of choice followed by other potential options. However, when the distal veins are unavailable, exhausted, or had calcified distal vessels are in need for alternative methods for surgical angioaccess. ${ }^{9}$ Autologous brachial artery fistulas are considered as a suitable option in these conditions. ${ }^{10}$ So, we have compared the two most commonly created autologous arteriovenous fistula.

In our study the age of the patient ranges from 15 to 81 years, with the mean age $56 \pm 16.12$ years. In the article by Lamicchane et al $^{11}$, the median age was 35.44 years which was 20 years younger than our patients. But in the study of Ahmed et al ${ }^{12}$, patient's age varied from 25 to 76 years with the mean age of $55 \pm 20$ years, which was similar to our study.

For the maturation of the fistula at least one and a half months is needed so that an autologous fistula can be used. The fistula should, therefore, preferably be created several months in advance of the anticipated need for dialysis. Most guidelines recommend a fistula should be placed at least 6 months before the anticipated start of HD treatments. ${ }^{13}$ But in our study only the 30 (7.5\%) patients created AV fistula before starting hemodialysis. It is very low compared to other studies. As much as 370 (92\%) patients were referred for creation of AV fistula after starting hemodialysis. This percentage is higher than that reported from Australia (28\%). ${ }^{14}$ This might be due to delay in diagnosis, delay in seeking medical advice due to lack of awareness of the patients, delay in referral to surgeons for the creation of the surgery. Internal jugular assay, subcutaneous permanent catheter and even the creation of AV fistula do not last long. After sometimes these assays do no longer work. Internal jugular assay works for about one and a half month, subcutaneous catheter work for about six months and AV fistula may work for a few years. So we have to create the AV fistula in different sites, once the already created fistula no longer works. In our study, re surgery was done in 39 (19.5\%) of RC groups while in BC group re surgery was done in $73(36.5 \%)$ cases due to failure of previously created AV fistula. But in other studies most stenosis AV fistula are treated with percutaneous transluminal angioplasty (PTA), which facility is lacking in our center. In the study of Bountourious, only $9 \%$ of the stenosis required surgical revision and only in $13 \%$ re surgery was done for the fistulas which failed permanently. ${ }^{15}$ Not all created AV fistulas are mature enough for the dialysis. Many factors like the site of the surgery, the size of the artery, vein, flow rate, comorbidities play the role for the maturation. ${ }^{16}$ In our study, there was a failure rate of $17 \%$ in RC AV fistula group and $8.5 \%$ in BC AV fistula group with overall failure rate of $12.75 \%$. In the study of Pogula et al the failure rate was in $18 \%$ RC AV fistula and $12.3 \%$ in BC AV fistula, with an overall failure rate of $15.15 \%$ which is similar to our study. Though AV fistulas are cheap and easy to construct, have excellent patency rates and require minimal maintenance by the patient and the health care staff, they can develop various complications. Most of them threaten the functionality of the fistula and some of them even pose an immediate ital. risk. It is important that all healthcare professionals who deal with patients on whom an AVF is performed should have through knowledge of the types, physiology, risks and treatment of these complications. The complications can be divided into acute and chronic, but since the follow up our patients were two months, we mainly discuss early complications. Bleeding is the most common acute complication. Spontaneous bleedings are not uncommon in uremic patients, in whom the primary mechanisms of hemostasis are compromised, including thrombocytopenia, platelet dysfunction and von Willebrand factor's changes. Bleeding sources have several causes. There are smaller sources, with no significant hemodynamic impact. These smaller sources are dermal, sub dermal or from the subcutaneous tissue. There are also larger sources, with a higher flow and a life threatening potential. They are usually found at the site of the anastomosis or a slipped vessel ligature and are accompanied by a hematoma. In the study of Susan et al the rate of thrombosis and bleeding was $8.5 \% .{ }^{17}$ This finding was similar to our study which was also the most common complication. There was no difference in the rate of bleeding between RC AVF and BC AVF. In RC AV fistula group 
Magar et al.: Comparative Study of Autologous Radiocephalic and Brachiocephalic Arteriovenous Fistula in Patients with End Stage Renal Disease

the bleeding rate was $10.5 \%$ and in BC AV fistula $9 \%$ with overall bleeding of $9.75 \%$. Hand edema is a relatively frequent, but usually transient complication in vascular access surgery. Venous hypertension occurs shortly after AVF creation, but it diminishes after collaterals develop and outflow improves. Outflow obstruction due to stenosis of a central vein provoked by a long term indwelling catheter or by neo intimal hyperplasia from the turbulent flow of the AVF also causes venous hypertension. If the hypertension does not subside, it is accompanied by the classic symptoms of a venous stasis syndrome: edema, pigmentation and ulceration .In the study of the edema in RC AVF was $1.23 \%$ and in BC AVF $5.79 \% .{ }^{18}$ But in contrast to this, limb edema was higher in the AVF group in our study. The edema in RC AVF was 3.5\% and in BC AVF was $8 \%$. We managed all limb edema conservatively.

Infection accounts for $20 \%$ of all AVF complications, which is ten times lower than the rate of infection of AVGs. ${ }^{19}$ Most AVF infections involve perivascular cellulitis, which manifests as localized erythema and edema and is usually easily treated. Much more serious is an infection associated with anatomical abnormalities, such as aneurysms, hematomas or abscesses, which require surgical excision and drainage..$^{20}$ In the study of Susan et al, the overall rate of wound infection was 3.4\% which was similar to our study where infection rate is $4.5 \%$. But there was a difference in wound infection rate between RC AVF and BC AVF. In the study of Khadatkar et al, the infection rate was $1.45 \%$ in RC AVF group and $1.23 \%$ in BC AVF group. In contrast, there was more infection in BC AVF group than in RC AVF group in our study having $6 \%$ and $3 \%$ respectively. We managed wound infection with regular dressing and antibiotics, when there was a gap we applied a secondary suture.

\section{LIMITATION}

The study was conducted only in single center, the follow up of the patients was of short duration so the findings could not be generalized in large population.

\section{CONCLUSION}

Though radio cephalic fistula is considered the first procedure of choice it's not always feasible to create it. The maturation and patency rate is higher in brachiocephalic arteriovenous fistula then radiocephalic arteriovenous fistula. But complications like limb edema and infection are slightly higher in brachiocephalic arteriovenous fistula.

\section{REFERENCES}

1. United States Renal Data System website. 2014 annual data report: epidemiology of kidney disease in the United States. www.usrds.org/2014/view/Default.aspx. Published 2014. Accessed July 5, 2015.

2. MacRae JM, Ahmed A, Johnson N, Levin A, Kiaii M. Central vein stenosis: a common problem in patients on hemodialysis. ASAIO J 2005; 51:77-81
3. Ravani P, Palmer SC, Oliver MJ, et al. Associations between hemodialysis access type and clinical clinical outcomes: a systematic review. J Am Soc Nephrol 2013; 24:465-473

4. Hakim R, Himmelfarb J. Hemodialysis access failure: a call to action. Kidney Int. 1998;54(4):1029-40.

5. Añel RL, Yevzlin AS. Vascular access and patient outcomes in hemodialysis: questions answered in recent literature. Artificial organs. 2003;27(3):237-41.

6. Feldman $\mathrm{HI}$, Kobrin S, Wasserstein A. Hemodialysis vascular access morbidity. J Am Soc Nephrol 1996; 7:35-523.

7. Allon M, Daurgidas J, Depner TA, Greene T, Ornt D, Schwab SJ. Effect of change in vascular access on patient mortality in hemodialysis patients. Am J Kidney Dis. 2006;47(3):469-477

8. Brescia MG, Cimino JE, Appel K. Chronic hemodialysis using venipuncture and a surgically created arteriovenous fistula. Engl J Med 1966; 275 N:1089-1092

9. Miller CD, Robbin ML, Allon M. Gender differences in outcomes of arteriovenous fistulas in hemodialysis patients. Kidney Int 2003; 63:346-352.

10. Tordoir JH, Dammers R, van der Sande FM. Upper extremity ischemia and hemodialysis vascular access. Eur J Vasc Endovasc Surg 2004; 27:1-5.

11. Lamichhane, D., Paudyal, S., Maskey, P., Maharjan, S., Joshi, A., Pandey, B., Shah, J., \& Jaiswal, V. Clinical profile and early outcome of arteriovenous fistula creation for haemodialysis: Integrated activity in a general surgery unit. Journal of Society of Surgeons of Nepal, 2007;18(2), 2-5.

12. Ahmed GM, Mansor MO, Elfaith M, Khalid KE,Ahmed ME. Outcomes of arteriovenous fistula for hemodialysis in Sudanese patients: Single-Centre experience. Saudi Journal of Kidney Disease and transplantation. 2012;23(1):152-157.

13. National Kidney Foundation. Retrive from http://www.kidney. org/professionals/kdoqi/guideline_uphd_pd_va/va_guide1.htm.

14. Jennings WC, Kindred MG, Broughan TA. Creating radiocephalic arteriovenous fistulas: technical and functional success. J Am Coll Surg. 2009;208:419.

15. Bountouris I, Kristmundsson T, Dias N, Zdanowski Z, Malina M. Is Repeat PTA of a Failing Hemodialysis Fistula Durable?. Int J Vasc Med. 2014;2014:369687.

16. Smith GE, Gohil R, Chetter IC. Factors affecting the patency of arteriovenous fistulas for dialysis access. J Vasc Surg 2012;55:849-55.

17. Susan J, Pawar B. Complications of arteriovenous fistula for haemodialysis access. Int Surg J 2018;5:439-44.

18. Khadatkar A, Mahakalkar C, Pradhan T, Bora A. Comparative analysis of radiocephalic versus brachiocephalic native arteriovenous fistula for hemodialysis in end stage renal disease. Int J Res Med Sci 2017;5:171-6.

19. Schild AF, Perez E, Gillaspie E, Seaver C, Livingstone J, Thibonnier $A$ : Arteriovenous fistula vs arteriovenous grafts: a retrospective review of 1700 consecutive vascular access cases. J Vasc Access 2008;9:231-235.

20. Saxena AK, Panhotra BR, Al-Mulhim AS: Vascular access related infections in hemodialysis patients. Saudi J Kidney Dis Transpl 2005;16:46-51. 